\title{
Effects of Rayleigh Waves on the Essential Spectrum in Perturbed Doubly Periodic Elliptic Problems
}

Bakharev, F. L.

2017-07

Bakharev , F L , Cardone , G , Nazarov , S A \& Taskinen , J 2017 , ' Effects of Rayleigh

Waves on the Essential Spectrum in Perturbed Doubly Periodic Elliptic Problems ', Integral

Equations and Operator Theory, vol. 88 , no. 3 , pp. 373-386 . https://doi.org/10.1007/s00020-017-2379-5

http://hdl.handle.net/10138/312496

https://doi.org/10.1007/s00020-017-2379-5

acceptedVersion

Downloaded from Helda, University of Helsinki institutional repository.

This is an electronic reprint of the original article.

This reprint may differ from the original in pagination and typographic detail.

Please cite the original version. 


\title{
Effects of Rayleigh waves on the essential spectrum in perturbed doubly periodic elliptic problems
}

\author{
F.Bakharev*, G.Cardoneł S.A.Nazarov ${ }^{\ddagger}$ J.Taskinen ${ }^{\S}$
}

November 8, 2018

\begin{abstract}
We give an example of a scalar second order differential operator in the plane with double periodic coefficients and describe its modification, which causes an additional spectral band in the essential spectrum. The modified operator is obtained by applying to the coefficients a mirror reflection with respect to a vertical or horizontal line. This change gives rise to Rayleigh type waves localized near the line. The results are proven using asymptotic analysis, and they are based on high contrast of the coefficient functions.
\end{abstract}

Keywords: periodic media, open waveguides, high contrast of coefficients, asymptotics, spectral bands.

MSC: Primary 35P05; Secondary 47A75.

\section{Introduction}

\subsection{Motivation.}

A satisfactory theory for spectral elliptic boundary-value problems in double periodic media containing open waveguides does not exist yet, and the topic contains a lot of unanswered questions. An open waveguide consists of a semi-infinite foreign inclusion, cf. Fig.1.1, and being a non-compact domain perturbation, it can in general change the essential spectrum of the problem, when compared to the corresponding problem on an intact domain without perturbation. This topic was studied for example in the recent paper [1], which contains a

\footnotetext{
*Chebyshev Laboratory, St. Petersburg State University, 14 th Line, 29b, Saint Petersburg, 199178 Russia; email: fbakharev@yandex.ru

†Università del Sannio, Department of Engineering, Corso Garibaldi, 107, 82100 Benevento, Italy; email: giuseppe.cardone@unisannio.it

${ }^{\ddagger}$ St. Petersburg State University, 198504, Universitetsky pr., 28, Stary Peterhof, Russia; Peter the Great St. Petersburg State Polytechnical University, Polytechnicheskaya ul., 29, St. Petersburg, 195251, Russia; Institute of Problems of Mechanical Engineering RAS, V.O., Bolshoj pr., 61, St. Petersburg, 199178, Russia; email: srgnazarov@yahoo.co.uk.

$\S$ University of Helsinki, Department of Mathematics and Statistics, P.O. Box 68, 00014 Helsinki, Finland; email: jari.taskinen@helsinki.fi.
} 
a)

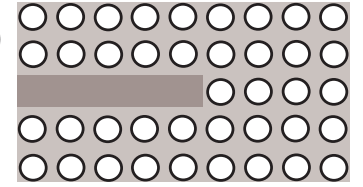

b)

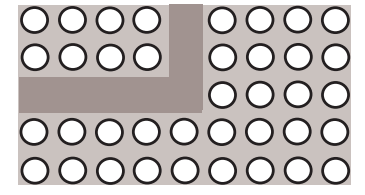

Figure 1.1: Semi-infinite (a) and angular (b) open waveguides in double-periodic planar domains.

complete description of the essential spectrum $\sigma_{\text {ess }}(\mathcal{T})$ for a large class of elliptic second order systems with Neumann boundary conditions, satisfying a Korn inequality. The following question 1 has arisen in the course of the investigation: is the formula

$$
\sigma_{\text {ess }}(\mathcal{T})=\sigma_{\text {ess }}^{-} \cup \sigma_{\text {ess }}^{+}
$$

valid for an elliptic problem in the union of two subdomains of the plane, which are contained in the lower and upper half-planes; here, $\sigma_{\text {ess }}^{-}$(respectively, $\sigma_{\text {ess }}^{+}$) denotes the essential spectrum of the corresponding problem in the lower (resp. upper) half-plane, and it is assumed that these two problems are periodic along the abscissa axis, but independently of each other? Obviously, the interesting aspect in this problem is to find a possible component of $\sigma_{\text {ess }}(\mathcal{T})$ which is not contained in the spectra of the problems in the subdomains.

As additional motivation of the problem we recall the case of the one-dimensional Schrödinger equation

$$
-\partial_{x}^{2} w(x)+V(x) w(x)=\lambda w(x), \quad x \in \mathbb{R}=(-\infty,+\infty)
$$

with the composite potential

$$
V(x)=V^{ \pm}(x) \text { for } \quad \pm x>\ell>0
$$

where $\partial_{x}=\partial / \partial x$ and $V^{ \pm}$are 1-periodic positive smooth functions; smoothness is assumed here for the sake of simplicity. The essential spectrum $\sigma_{\text {ess }}$ of the problem (1.2) is just the union of the spectra $\sigma_{\text {ess }}^{ \pm}$of the differential operators $-\partial_{x}^{2}+V^{ \pm}$with periodic coefficients in the whole axis $\mathbb{R}$. This fact is evident because the equation can be reformulated as system of ordinary differential equations

$$
-\partial_{x}^{2} w^{ \pm}(x)+V(x) w^{ \pm}(x)=\lambda w^{ \pm}(x), \quad x \in \mathbb{R}_{ \pm}=\{x \in \mathbb{R}: \pm x>0\},
$$

with transmission conditions

$$
w^{+}(+0)=w^{-}(-0), \quad \partial_{x} w^{+}(+0)=\partial_{x} w^{-}(-0) .
$$

Indeed, according to (1.3), the essential spectrum of (1.4) with Dirichlet conditions $w^{ \pm}(0)=0$ is nothing but $\sigma_{\text {ess }}^{ \pm}$, while the system (1.4), (1.5) differs from the couple of the Dirichlet problems in $\mathbb{R}_{ \pm}$by a localized perturbation (it can be interpreted as a compact perturbation).

Coming back to the problem (1.1), the above described argument based on a compact perturbation works no longer, since the interface $\partial \mathbb{R}_{ \pm}^{2}$, where $\mathbb{R}_{ \pm}^{2}=\left\{\left(x_{1}, x_{2}\right) \in \mathbb{R}^{2}: \pm x_{1} \geq\right.$

\footnotetext{
${ }^{1}$ The question has been asked by a referee of the paper [1], among others
} 


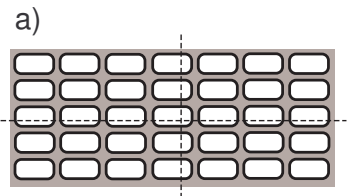

c)

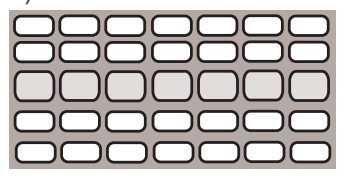

b)

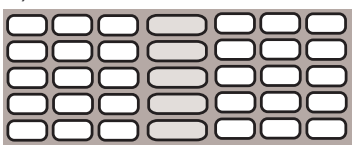

d)

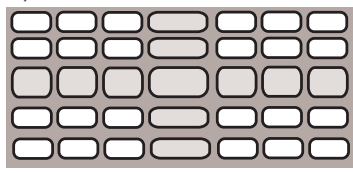

Figure 1.2: Double-periodic planar domain (a) and composite domains (b-d) created by mirror reflections.

$0\}$, is infinite. However, there is no easy, satisfactory counterexample to the relationship (1.1), if the natural requirements like smoothness of the coefficient are to be satisfied; this prevents answering the question directly by using classical Rayleigh waves [2], [3] in elasticity and their generalizations, see [4].

In the present paper we give examples of elliptic scalar equations with smooth double periodic coefficients, which have the following property: if the plane is divided along dotted lines in Fig. 1.2 a) and the left or upper half-plane is doubled by using mirror reflection, Fig. 1.2.b) or c), the new elliptic problem gains essential spectrum $\sigma_{\text {ess }}(\mathcal{T})$ with at least one additional spectral band in comparison with the original spectrum $\sigma_{\text {ess }}\left(\mathcal{T}^{0}\right)$ of the double periodic problem. This main result of our paper is formulated in Theorem 4 in Section 3 , We also mention that the result of [1] is proven in two steps: the first consists of finding a singular Weyl sequence at any point $\lambda \in \sigma_{\text {ess }}(\mathcal{T})$ for the problem operator $\mathcal{T}$ and the second of the construction of a (right) parametrix for the problem with any $\lambda \notin \sigma_{\text {ess }}(\mathcal{T})$. It has been asked, if it is possible to avoid the quite technical and cumbersome construction of the parametrix, like it has been done in the case of the one-dimensional Schrödinger equation. The present paper also demonstrates the complications in this respect.

To fulfill the task, we employ an elegant formulation of [5], see also [6, 7, 8], on the detection of spectral gaps in scalar problems, where the coefficients of the differential operator have high contrast. However, we were not able to apply these results directly, and modifications are presented in Section 3 in order to satisfy all natural assumptions. In particular, we find a way to keep the infinite smoothness of the coefficients; note that in [5, 6, 7, 8] the coefficients have to be piecewise constant. In particular, as is drafted in Fig.1.2.a), the massive hard parts of the double periodic medium are separated by thin, soft "mortar" like in hand-made masonry (similar structure appears also in natural quarzites). Compared with the citations, especially [8] where a similar geometric structure was employed for a different purpose, we use quite a different scheme of asymptotic analysis, which also leads to new asymptotic results about the purely periodic case in Section 2 (Theorem 1). In order to clarify the proof of our main result we will accept some simplifying assumptions. Possible generalizations will be discussed in Sect. 4. 


\subsection{Purely periodic medium.}

We now describe the double periodic elliptic second order partial differential equation which will be investigated in Section 2, The main example of the failure of the equality (1.1) for the composite medium $\mathbb{R}_{+}^{2} \cup \mathbb{R}_{-}^{2}$ will be constructed in Section 3 .

We define the period cell as the rectangle $Q=\left(-\ell_{1}, \ell_{1}\right) \times\left(-\ell_{2}, \ell_{2}\right)$ with $\ell_{1} \geq \ell_{2}>0$. For $\varepsilon \in\left(0, \ell_{2}\right)$ we introduce a smaller rectangle $Q_{\varepsilon}=\left(-\ell_{1}+\varepsilon, \ell_{1}-\varepsilon\right) \times\left(-\ell_{2}+\varepsilon, \ell_{2}-\varepsilon\right)$. Let us also define a family of translated domains

$$
Q_{\varepsilon}(\alpha)=\left\{x=\left(x_{1}, x_{2}\right):\left(x_{1}-2 \ell_{1} \alpha_{1}, x_{2}-2 \ell_{2} \alpha_{2}\right) \in Q_{\varepsilon}\right\}
$$

where $\alpha=\left(\alpha_{1}, \alpha_{2}\right) \in \mathbb{Z}^{2}$ and $\mathbb{Z}=\{0, \pm 1, \pm 2, \ldots\}$.

We consider the spectral problem

$$
-\operatorname{div}\left(a^{\varepsilon}(x) \nabla_{x} u^{\varepsilon}(x)\right)=\lambda^{\varepsilon} u^{\varepsilon}(x), \quad x \in \mathbb{R}^{2},
$$

where $\nabla_{x}$ is the gradient in the variable $x$ and $\lambda^{\varepsilon}$ is a spectral parameter. The function $a^{\varepsilon}$ is smooth and $2 \ell_{j}$-periodic in $x_{j}$ such that

$$
a^{\varepsilon}(x)=1, \quad x \in Q_{2 \varepsilon}, \quad a^{\varepsilon}(x)=\varepsilon^{2 \gamma}, \quad x \in Q \backslash \overline{Q_{\varepsilon}},
$$

and $a^{\varepsilon}(x) \in\left(\varepsilon^{2 \gamma}, 1\right]$ if $x \in Q_{\varepsilon} \backslash Q_{2 \varepsilon}$, where $\gamma \in(1 / 2,1)$ is a fixed parameter.

The variational formulation of the problem (1.6) reads as

$$
\left(a^{\varepsilon} \nabla_{x} u^{\varepsilon}, \nabla_{x} v\right)_{\mathbb{R}^{2}}=\lambda^{\varepsilon}\left(u^{\varepsilon}, v\right)_{\mathbb{R}^{2}}, \quad v \in H^{1}\left(\mathbb{R}^{2}\right)
$$

where $(f, g)_{\Omega}$ stands for the usual (complex valued) inner product in $L^{2}(\Omega)$ for a domain $\Omega \subset \mathbb{R}^{2}$. We denote the standard Sobolev space by $H^{1}(\Omega)$. The sesquilinear form on the left of (1.8) is positive and closed in $H^{1}\left(\mathbb{R}^{2}\right)$ and consequently (see [9, Ch. 10], [10, Thm. VIII.5]) our problem can be rewritten as an abstract operator equation $\mathcal{T}^{0}(\varepsilon) u^{\varepsilon}=\lambda^{\varepsilon} u^{\varepsilon}$, where $\mathcal{T}^{0}(\varepsilon)$ is an unbounded positive self-adjoint operator in Hilbert space $L^{2}\left(\mathbb{R}^{2}\right)$ with domain $\mathcal{D}\left(\mathcal{T}^{0}(\varepsilon)\right)=H^{2}\left(\mathbb{R}^{2}\right)$, and thus the spectrum $\sigma\left(\mathcal{T}^{0}(\varepsilon)\right)$ is a subset of the semi-axis $\overline{\mathbb{R}_{+}}=$ $[0,+\infty)$. The embedding $H^{1}\left(\mathbb{R}^{2}\right) \subset L^{2}\left(\mathbb{R}^{2}\right)$ is not compact, hence the essential spectrum $\sigma_{\text {ess }}\left(\mathcal{T}^{0}(\varepsilon)\right)$ is not empty.

\section{Asymptotic analysis of the spectrum of the purely periodic problem}

\subsection{FBG-transform and model problem in the period cell.}

The Floquet-Bloch-Gelfand-(FBG-)transform, see [11] and also [12, 13, 14, 15], converts the differential equation (1.6) into the following problem with quasiperiodic boundary conditions in the period cell $Q$,

$$
\begin{aligned}
& -\operatorname{div}\left(a^{\varepsilon}(x) \nabla_{x} U^{\varepsilon}(x ; \eta)\right)=\Lambda^{\varepsilon}(\eta) U^{\varepsilon}(x ; \eta), \quad x \in Q, \\
& \left.U^{\varepsilon}(x ; \eta)\right|_{x_{j}=\ell_{j}}=\left.e^{i \eta_{j}} U^{\varepsilon}(x ; \eta)\right|_{x_{j}=-\ell_{j}}, \quad\left|x_{3-j}\right|<\ell_{3-j}, \\
& \left.\partial_{j} U^{\varepsilon}(x ; \eta)\right|_{x_{j}=\ell_{j}}=\left.e^{i \eta_{j}} \partial_{j} U^{\varepsilon}(x ; \eta)\right|_{x_{j}=-\ell_{j}}, \quad\left|x_{3-j}\right|<\ell_{3-j},
\end{aligned}
$$


where $j=1,2, \partial_{j}=\partial / \partial x_{j}$ and $\eta=\left(\eta_{1}, \eta_{2}\right)$ is the Floquet parameter in the closed rectangle $R=\left[0, \pi \ell_{1}^{-1}\right] \times\left[0, \pi \ell_{2}^{-1}\right]$. In the sequel we do not always display the dependence on $\eta$ explicitly. The problem has the variational formulation

$$
\left(a^{\varepsilon} \nabla_{x} U^{\varepsilon}, \nabla_{x} V\right)_{Q}=\Lambda^{\varepsilon}(\eta)\left(U^{\varepsilon}, V\right)_{Q} \quad \forall V \in H_{\eta}^{1}(Q),
$$

where $H_{\eta}^{1}(Q)$ is the Sobolev space of functions satisfying the conditions (2.2). The bilinear form on the left of (2.4) is positive and closed in $H^{1}(Q)$. Hence, since the embedding $H^{1}(Q) \subset L^{2}(Q)$ is compact, the spectrum of the problem (2.4) or (2.1)-(2.3) is discrete and turns into the monotone unbounded sequence

$$
0 \leq \Lambda_{1}^{\varepsilon}(\eta) \leq \Lambda_{2}^{\varepsilon}(\eta) \leq \ldots \leq \Lambda_{k}^{\varepsilon}(\eta) \leq \ldots \rightarrow+\infty,
$$

and the corresponding eigenfunctions $U_{1}^{\varepsilon}(\cdot ; \eta), U_{2}^{\varepsilon}(\cdot ; \eta), \ldots$ can be subject to the normalization and orthogonality conditions

$$
\left(U_{j}^{\varepsilon}, U_{k}^{\varepsilon}\right)_{Q}=\delta_{j, k}, \quad j, k \in \mathbb{N},
$$

where $\delta_{j, k}$ is the Kronecker symbol.

The functions $R \ni \eta \mapsto \Lambda_{k}^{\varepsilon}(\eta)$ are continuous and $\pi \ell_{j}^{-1}$-periodic in the variable $\eta_{j}$, cf. [17, Ch.VII]. As was verified for example in [13, 14, 15], the spectrum of the problem (1.6) or (1.8) has band-gap structure,

$$
\sigma\left(\mathcal{T}^{0}(\varepsilon)\right)=\bigcup_{k \in \mathbb{N}} \beta_{k}^{\varepsilon}, \quad \beta_{k}^{\varepsilon}=\left\{\Lambda_{k}^{\varepsilon}(\eta) \mid \eta \in R\right\},
$$

where the sets $\beta_{k}^{\varepsilon}$ are closed finite intervals. Our actual objective is to describe the sets in (2.7) asymptotically as $\varepsilon \rightarrow+0$.

\subsection{Limit model problem and theorem on asymptotics.}

We will next study the relation of the eigenvalues (2.5) and the spectrum of the so-called limit problem

$$
\begin{aligned}
& -\Delta_{x} w(x)=\mu w(x), \quad x \in Q, \\
& \partial_{n} w(x)=0, \quad x \in \partial Q,
\end{aligned}
$$

where $\Delta_{x}$ is the Laplace operator in the variables $x$ and $\partial_{n}$ is the outward normal derivative. The problem (2.8), (2.9) can be solved explicitly. Its spectrum consists of the eigenvalue sequence $\left\{\mu_{n}\right\}_{n \in \mathbb{N}}=\left\{\frac{\pi^{2}}{4}\left(j^{2} \ell_{1}^{-2}+k^{2} \ell_{2}^{-2}\right)\right\}_{j, k \in \mathbb{N} \cup\{0\}}$, which is indexed taking into account multiplicities such that

$$
0=\mu_{1}<\mu_{2} \leq \mu_{3} \leq \ldots \leq \mu_{n} \leq \ldots \rightarrow+\infty .
$$

To simplify forthcoming calculations we assume that $\ell_{1}^{2} \ell_{2}^{-2}$ is not rational. This guarantees that all eigenvalues in (2.10) are simple. The corresponding eigenfunctions

$$
w_{n}(x)=c_{j k} \cos \left(\pi\left(2 \ell_{1}\right)^{-1} j\left(x_{1}+\ell_{1}\right)\right) \cos \left(\pi\left(2 \ell_{2}\right)^{-1}\left(x_{2}+\ell_{2}\right)\right),
$$


with $c_{j k}^{2}=\left(1+\delta_{j, 0}\right)\left(1+\delta_{k, 0}\right)\left(\ell_{1} \ell_{2}\right)^{-1}$ satisfy the normalization and orthogonality conditions $\left(w_{n}, w_{m}\right)_{Q}=\delta_{n, m}, n, m \in \mathbb{N}$.

We note that the problem (2.8)-(2.9) has the variational form

$$
\left(\nabla_{x} w, \nabla_{x} v\right)_{Q}=\mu(w, v)_{Q} \quad \forall v \in H^{1}(Q) .
$$

The main result in Section 2 is the following assertion, the proof of which will be completed in Section 2.4.

Theorem 1 For every $n \in \mathbb{N}$, there exist positive $\varepsilon_{n}$ and $c_{n}$ such that the eigenvalues (2.5) and (2.10) are related by

$$
\left|\Lambda_{n}^{\varepsilon}(\eta)-\mu_{n}\right| \leq c_{n} \varepsilon^{\gamma-1 / 2} \quad \text { for } \quad \varepsilon \in\left(0, \varepsilon_{n}\right] .
$$

\subsection{Convergence theorem and identification of spectral gaps.}

Let us denote by $\mu_{n}^{D}$ the $n$th eigenvalue (ordered as in (2.10) ) for the Dirichlet problem in $Q$, consisting of the differential equation (2.8) and the boundary condition $w=0$ on $\partial Q$ instead of (2.9) . By the max-min principle, see e.g., [9, Thm. 10.2.2], [16, Thm. XIII 1,2] we readily

conclude that $\Lambda_{n}^{\varepsilon}(\eta) \leq \mu_{n}^{D}$. Then, for the eigenfunction $U_{n}^{\varepsilon}$ of the problem (2.1)-(2.3), we have

$$
\begin{aligned}
& \left\|\nabla_{x} U_{n}^{\varepsilon} ; L^{2}\left(Q_{2 \varepsilon}\right)\right\|^{2}+\left\|\sqrt{a^{\varepsilon}} \nabla_{x} U_{n}^{\varepsilon} ; L^{2}\left(Q_{\varepsilon} \backslash Q_{2 \varepsilon}\right)\right\|^{2} \\
& +\varepsilon^{2 \gamma}\left\|\nabla_{x} U_{n}^{\varepsilon} ; L^{2}\left(Q \backslash Q_{\varepsilon}\right)\right\|^{2} \leq \mu_{n}^{D} .
\end{aligned}
$$

Denoting the coordinate dilation by $A_{\varepsilon} x=\left(\left(1-2 \varepsilon \ell_{1}^{-1}\right) x_{1},\left(1-2 \varepsilon \ell_{2}^{-1}\right) x_{2}\right)$, the $H^{1}(Q)$-norm of the function

$$
\mathbf{U}_{n}^{\varepsilon}(x ; \eta)=U_{n}^{\varepsilon}\left(A_{\varepsilon} x ; \eta\right)
$$

is uniformly bounded with respect to $\varepsilon \in(0,1]$ and $\eta \in R$. Hence, for some positive sequence $\left\{\varepsilon_{p}\right\}_{p \in \mathbb{N}}$ converging to 0 , we have

$$
\Lambda_{n}^{\varepsilon_{p}}(\eta) \rightarrow \Lambda_{n}^{0}(\eta), \quad \mathbf{U}_{n}^{\varepsilon_{p}} \rightarrow \mathbf{U}_{n}^{0} \quad \text { as } p \rightarrow \infty,
$$

where the latter convergence happens weakly in $H^{1}(Q)$ and strongly in $L^{2}(Q)$.

Let $v^{0}$ be an arbitrary smooth function in $\bar{Q}$ and set

$$
v^{\varepsilon}(x)=X^{\varepsilon}(x) v^{0}\left(A_{\varepsilon}^{-1} x\right),
$$

where $X^{\varepsilon}: Q \rightarrow[0,1]$ is a smooth cut-off function such that

$$
X^{\varepsilon}=1 \text { in } Q_{\varepsilon}, \quad X^{\varepsilon}=0 \text { in } Q \backslash Q_{\varepsilon / 2}, \quad \text { and }\left|\nabla_{x} X^{\varepsilon}\right| \leq C_{X} \varepsilon^{-1} \text { in } Q .
$$

Since $X^{\varepsilon}=0$ near $\partial Q$, the function (2.17) satisfies the quasiperiodicity conditions (2.2) and therefore can be inserted into the integral identity (2.4):

$$
\left(a^{\varepsilon} \nabla_{x} U_{n}^{\varepsilon}, \nabla_{x} v^{\varepsilon}\right)_{Q}=\Lambda_{n}^{\varepsilon}(\eta)\left(U_{n}^{\varepsilon}, v^{\varepsilon}\right)_{Q}
$$


Here we have

$$
\Lambda_{n}^{\varepsilon}(\eta)\left(U_{n}^{\varepsilon}, v^{\varepsilon}\right)_{Q} \rightarrow \Lambda_{n}^{0}(\eta)\left(\mathbf{U}_{n}^{0}, v^{0}\right)_{Q} \quad \text { as } \varepsilon \rightarrow 0,
$$

because, first,

$$
\begin{aligned}
& \left(U_{n}^{\varepsilon}, v^{\varepsilon}\right)_{Q_{2 \varepsilon}}=\int_{Q_{2 \varepsilon}} \mathbf{U}_{n}^{\varepsilon}\left(A_{\varepsilon}^{-1} x\right) \overline{v^{0}\left(A_{\varepsilon}^{-1} x\right)} d x \\
= & \left(1-2 \varepsilon \ell_{1}^{-1}\right)\left(1-2 \varepsilon \ell_{2}^{-1}\right)\left(\mathbf{U}_{n}^{\varepsilon}, v^{0}\right)_{Q} \rightarrow\left(\mathbf{U}_{n}^{0}, v^{0}\right)_{Q}
\end{aligned}
$$

and, second,

$$
\left|\left(U_{n}^{\varepsilon}, v^{\varepsilon}\right)_{Q \backslash Q_{2 \varepsilon}}\right| \leq c\left(v^{0}\right)\left\|U_{n}^{\varepsilon} ; L^{2}(Q)\right\|\left|Q \backslash Q_{2 \varepsilon}\right|^{1 / 2} \leq c_{n}\left(v^{0}\right) \sqrt{\varepsilon},
$$

where we take into account the normalization condition (2.6), the boundedness of the function $v^{0}$ and the area $\left|Q \backslash Q_{2 \varepsilon}\right|=O(\varepsilon)$ of the integration domain $q \backslash Q_{2 \varepsilon}$. A transformation similar to (2.21) shows that

$$
\left(a^{\varepsilon} \nabla_{x} U_{n}^{\varepsilon}, \nabla_{x} v^{\varepsilon}\right)_{Q_{2 \varepsilon}} \rightarrow\left(\nabla_{x} \mathbf{U}_{n}^{0}, \nabla_{x} v^{0}\right)_{Q}
$$

because $a^{\varepsilon}=1$ on $Q_{2 \varepsilon}$. Moreover,

$$
\begin{aligned}
& \left(a^{\varepsilon} \nabla_{x} U_{n}^{\varepsilon}, \nabla_{x} v^{\varepsilon}\right)_{Q_{\varepsilon} \backslash Q_{2 \varepsilon}}=\left(a^{\varepsilon} \nabla_{x} U_{n}^{\varepsilon}, \nabla_{x}\left(v^{0} \circ A_{\varepsilon}^{-1}\right)\right)_{Q_{\varepsilon} \backslash Q_{2 \varepsilon}} \\
\leq & \left\|\sqrt{a^{\varepsilon}} \nabla_{x} U_{n}^{\varepsilon} ; L^{2}\left(Q_{\varepsilon} \backslash Q_{2 \varepsilon}\right)\right\|\left\|\sqrt{a^{\varepsilon}} \nabla_{x}\left(v^{0} \circ A_{\varepsilon}^{-1}\right) ; L^{2}\left(Q_{\varepsilon} \backslash Q_{2 \varepsilon}\right)\right\| \\
\leq & \mu_{n}^{D} c_{n}(v) \varepsilon^{1 / 2} .
\end{aligned}
$$

Finally,

$$
\begin{aligned}
\left(a^{\varepsilon} \nabla_{x} U_{n}^{\varepsilon}, \nabla_{x} v^{\varepsilon}\right)_{Q \backslash Q_{\varepsilon}} & \leq \varepsilon^{2 \gamma}\left\|\nabla_{x} U_{n}^{\varepsilon} ; L^{2}(Q)\right\|\left\|\nabla_{x} v^{\varepsilon} ; L^{2}\left(Q \backslash Q_{\varepsilon}\right)\right\| \\
& \leq \sqrt{\mu_{n}^{D}} \varepsilon^{\gamma} C_{X} \varepsilon^{-1} c_{v} \varepsilon^{1 / 2} \leq C_{n}(v) \varepsilon^{\gamma-1 / 2} .
\end{aligned}
$$

Here, we have used (2.14) to estimate the norm of $\nabla_{x} U_{n}^{\varepsilon}$ and (2.17), (2.18) for $\nabla_{x} v^{\varepsilon}$. Since $\gamma>1 / 2$, formulas (2.22) $-(2.24)$ imply

$$
\left(a^{\varepsilon} \nabla_{x} U_{n}^{\varepsilon}, \nabla_{x} v^{\varepsilon}\right)_{Q} \rightarrow\left(\nabla_{x} \mathbf{U}_{n}^{0}, \nabla_{x} v^{0}\right)_{Q} \quad \text { as } \varepsilon \rightarrow 0 .
$$

We formulate the following result of our calculations.

Proposition 2 For every $n \in \mathbb{N}$, the limit $\lambda_{n}^{0}(\eta)$ in (2.16) is an eigenvalue of the Neumann problem (2.8), (2.9), and $\mathbf{U}_{n}^{0}$ in (2.16) is the corresponding eigenfunction with normalization $\left\|\mathbf{U}_{n}^{0} ; L^{2}(Q)\right\|=1$.

Proof The fact that $\left(\lambda_{n}^{0}(\eta), \mathbf{U}_{n}^{0}\right)$ is the claimed eigenpair follows from the variational formulation (2.12), the arbitrariness of the choice of $v^{0}$, the density of smooth functions in the Sobolev space, the property (2.19), and the proven convergence in (2.20), (2.25).

It suffices to verify the normalization of $\mathbf{U}_{n}^{0}$. To this end, we use the inequality

$$
\left\|U_{n}^{\varepsilon} ; L^{2}\left(Q \backslash Q_{2 \varepsilon}\right)\right\|^{2} \leq c\left(\varepsilon^{2}\left\|\nabla_{x} U_{n}^{\varepsilon} ; L^{2}\left(Q \backslash Q_{2 \varepsilon}\right)\right\|^{2}+\varepsilon\left\|U_{n}^{\varepsilon} ; L^{2}\left(\partial Q_{2 \varepsilon}\right)\right\|^{2}\right)
$$




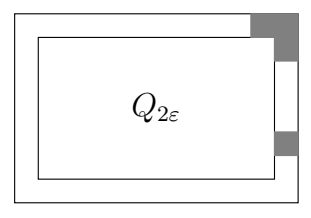

a)

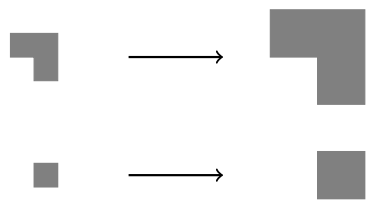

b)

c)

Figure 2.1: The division of the thin frame.

which can be derived by covering the thin frame $Q \backslash Q_{2 \varepsilon}$ with sets of diameter $O(\varepsilon)$, see Fig.2.1.a),b), stretching local coordinate systems by a factor of magnitude $\varepsilon^{-1}$ and applying standard trace inequalities in two kinds of sets, see Fig.(2.1.c). For the right hand side of (2.26) we use the inequalities

$$
\begin{aligned}
\left\|U_{n}^{\varepsilon} ; L^{2}\left(\partial Q_{2 \varepsilon}\right)\right\|^{2} & \leq C\left(\left\|\nabla_{x} U_{n}^{\varepsilon} ; L^{2}\left(Q_{2 \varepsilon}\right)\right\|^{2}+\left\|U_{n}^{\varepsilon} ; L^{2}\left(Q_{2 \varepsilon}\right)\right\|^{2}\right) \\
& \leq C\left(\Lambda_{n}^{\varepsilon}(\eta)+1\right)\left\|U_{n}^{\varepsilon} ; L^{2}(Q)\right\|^{2} \leq C_{n}, \\
\left\|\nabla_{x} U_{n}^{\varepsilon} ; L^{2}\left(Q \backslash Q_{2 \varepsilon}\right)\right\|^{2} & \leq \varepsilon^{-2 \gamma}\left\|\sqrt{a^{\varepsilon}} \nabla_{x} U_{n}^{\varepsilon} ; L^{2}(Q)\right\|^{2} \leq C_{n} \varepsilon^{-2 \gamma},
\end{aligned}
$$

which are based on the estimate (2.14) and the definition of $a^{\varepsilon}$. As a consequence of (2.6), (2.26) and $\gamma<1$ we get the desired normalization

$$
1=\left\|U_{n}^{\varepsilon} ; L^{2}(Q)\right\|^{2}=\left\|U_{n}^{\varepsilon} ; L^{2}\left(Q_{2 \varepsilon}\right)\right\|^{2}+O\left(\varepsilon^{2(1-\gamma)}+\varepsilon\right) \rightarrow\left\|\mathbf{U}_{n}^{0} ; L^{2}(Q)\right\|^{2} .
$$

Formula (2.15) passes the strong convergence in $L^{2}(Q)$, see (2.16), from $\mathbf{U}_{n}^{\varepsilon}$ to the eigenfunction $U_{n}^{\varepsilon}$ itself.

Since the limits of the eigenvalues in (2.5) belong to the set $\left\{\mu_{n}\right\}_{n \in \mathbb{N}}$ of isolated points, one finds any prescribed number of open gaps in the spectrum (2.7) by assuming the parameter $\varepsilon$ to be sufficiently small (a similar conclusion on the number of spectral bands is made in [7] for the problem introduced in [5], and the same conclusion can be made in [8], too).

\subsection{Asymptotics and estimates for spectral bands.}

In the Hilbert space $\mathcal{H}^{\varepsilon}=H_{\eta}^{1}(Q)$ we introduce the scalar product

$$
\langle u, v\rangle_{\varepsilon}=\left(a^{\varepsilon} \nabla_{x} u, \nabla_{x} v\right)_{Q}+(u, v)_{Q}
$$

and the positive, symmetric, continuous (consequently, self-adjoint) operator $\mathcal{K}^{\varepsilon}$,

$$
\left\langle\mathcal{K}^{\varepsilon} u, v\right\rangle_{\varepsilon}=(u, v)_{Q} \quad \forall u, v \in \mathcal{H}^{\varepsilon} .
$$

Comparing (2.27), (2.28) with (2.4), we see that the variational formulation of the problem (2.1)-(2.3) is equivalent to the abstract equation

$$
\mathcal{K}^{\varepsilon} u^{\varepsilon}=\kappa^{\varepsilon} u^{\varepsilon} \text { in } \mathcal{H}^{\varepsilon}
$$

with the new spectral parameter

$$
\kappa^{\varepsilon}=\left(1+\Lambda^{\varepsilon}\right)^{-1} .
$$


The well-known formula

$$
\operatorname{dist}\left(k^{\varepsilon}, \sigma\left(\mathcal{K}^{\varepsilon}\right)\right)=\left\|\left(\mathcal{K}^{\varepsilon}-k^{\varepsilon}\right)^{-1} ; \mathcal{H}^{\varepsilon} \rightarrow \mathcal{H}^{\varepsilon}\right\|^{-1}
$$

follows from the spectral decomposition of the resolvent $\left(\mathcal{K}^{\varepsilon}-k^{\varepsilon}\right)^{-1}$, e.g, [9, Ch 6, §3], [18, Thm. 12.23]. To estimate the operator norm of the resolvent at the "interesting" point $k^{\varepsilon}=\left(1+\mu_{n}\right)^{-1}$, we set $\mathcal{W}^{\varepsilon}=\left\|w^{\varepsilon} ; \mathcal{H}^{\varepsilon}\right\|^{-1} w^{\varepsilon}$, where $w^{\varepsilon}(x)=X^{\varepsilon}(x) w_{n}\left(A_{\varepsilon}^{-1} x\right)$, $\mu_{n}$ is an eigenvalue of the problem (2.8), (2.9), and the corresponding eigenfunction $w_{n}$ is extended to the exterior of $Q$ by its formula (2.11). We have

$$
\begin{aligned}
& \left\|\mathcal{K}^{\varepsilon} \mathcal{W}^{\varepsilon}-k^{\varepsilon} \mathcal{W}^{\varepsilon} ; \mathcal{H}^{\varepsilon}\right\|=\sup \left|\left\langle\mathcal{K}^{\varepsilon} \mathcal{W}^{\varepsilon}-k^{\varepsilon} \mathcal{W}^{\varepsilon}, v\right\rangle_{\varepsilon}\right| \\
= & \left(1+\mu_{n}\right)^{-1}\left\|w^{\varepsilon} ; \mathcal{H}^{\varepsilon}\right\|^{-1} \sup \left|\left(a^{\varepsilon} \nabla_{x} w^{\varepsilon}, \nabla_{x} v\right)_{Q}-\mu_{n}\left(w^{\varepsilon}, v\right)_{Q}\right|,
\end{aligned}
$$

where the supremum is computed over the unit ball in $\mathcal{H}^{\varepsilon}$. The expression inside the modulus signs in (2.31) equals the sum of the following terms:

$$
\begin{aligned}
& I_{1}^{\varepsilon}=\left(\nabla_{x}\left(w_{n} \circ A_{\varepsilon}^{-1}\right), \nabla_{x} v\right)_{Q_{2 \varepsilon}}-\mu_{n}\left(w_{n} \circ A_{\varepsilon}^{-1}, v\right)_{Q_{2 \varepsilon}}, \\
& I_{2}^{\varepsilon}=\left(\sqrt{a^{\varepsilon}} \nabla_{x}\left(w_{n}^{\varepsilon} \circ A_{\varepsilon}^{-1}\right), \sqrt{a^{\varepsilon}} \nabla_{x} v\right)_{Q_{\varepsilon} \backslash Q_{2 \varepsilon}} \\
& \quad-\mu_{n}\left(w_{n}^{\varepsilon} \circ A_{\varepsilon}^{-1}, v\right)_{Q_{\varepsilon} \backslash Q_{2 \varepsilon}}, \\
& I_{3}^{\varepsilon}=\varepsilon^{2 \gamma}\left(\nabla_{x}\left(X_{\varepsilon} w_{n} \circ A_{\varepsilon}^{-1}\right), \nabla_{x} v\right)_{Q \backslash Q_{\varepsilon}}-\mu_{n}\left(X_{\varepsilon} w_{n} \circ A_{\varepsilon}^{-1}, v\right)_{Q \backslash Q_{\varepsilon}} .
\end{aligned}
$$

Stretching variables and taking (2.12) into account yield

$$
\left|I_{1}^{\varepsilon}\right|=\left|2 \varepsilon \ell_{2}^{-1}\left(\partial_{x_{1}} w_{n}^{\varepsilon}, \partial_{x_{1}}\left(v \circ A_{\varepsilon}\right)\right)_{Q}+2 \varepsilon \ell_{1}^{-1}\left(\partial_{x_{2}} w_{n}^{\varepsilon}, \partial_{x_{2}}\left(v \circ A_{\varepsilon}\right)\right)_{Q}\right| \leq c_{n} \varepsilon .
$$

Since $w_{n}$ is a smooth function, we have

$$
\left|I_{2}^{\varepsilon}\right| \leq c_{n}\left|Q_{\varepsilon} \backslash Q_{2 \varepsilon}\right|\left\|v ; \mathcal{H}^{\varepsilon}\right\| \leq c_{n} \varepsilon^{1 / 2}
$$

In the same way, taking into account the bound for $\nabla_{x} X_{\varepsilon}$ in (2.18), we obtain

$$
\left|I_{3}^{\varepsilon}\right| \leq C_{n}\left(\varepsilon^{\gamma} \varepsilon^{-1} \varepsilon^{1 / 2}\left\|\varepsilon^{\gamma} \nabla_{x} v ; L^{2}\left(Q \backslash Q_{\varepsilon}\right)\right\|+\varepsilon^{1 / 2}\left\|v ; L^{2}(Q)\right\|\right) \leq c_{n} \varepsilon^{\gamma-1 / 2}
$$

These estimates for the terms in (2.32) and (2.31) show that the norm of the resolvent $\left(\mathcal{K}^{\varepsilon}-k^{\varepsilon}\right)^{-1}$ exceeds $c_{n} \varepsilon^{-(\gamma-1 / 2)}$ for some constant $c_{n}>0$. Thus, in view of the relation (2.30), the interval

$$
\left[k^{\varepsilon}-c_{n} \varepsilon^{\gamma-1 / 2}, k^{\varepsilon}+c_{n} \varepsilon^{\gamma-1 / 2}\right]
$$

contains an eigenvalue of $\mathcal{K}^{\varepsilon}$. Furthermore, the identity (2.29) shows that at least one eigenvalue in (2.5) falls into the short segment $\Upsilon_{n}=\left[\mu_{n}-C_{n} \varepsilon^{\gamma-1 / 2}, \mu_{n}+C_{n} \varepsilon^{\gamma-1 / 2}\right]$ with some $C_{n}>0$ (recall that $\gamma>1 / 2$ ). To conclude that this eigenvalue is unique and coincides with $\Lambda_{n}^{\varepsilon}(\eta)$, we use Proposition 2.2. If one of the segments $\Upsilon_{1}, \Upsilon_{2}, \ldots, \Upsilon_{n}$ includes two eigenvalues, then $\Lambda_{n+1}^{\varepsilon}(\eta)$ does not exceed $\mu_{n}+C_{n} \varepsilon^{\gamma-1 / 2}$ and, therefore, converges to $\Lambda_{n+1}^{0}(\eta) \leq \mu_{n}$, while the limit $U_{n+1}^{0}$ of the corresponding eigenfunction is orthogonal to $w_{1}, w_{2}, \ldots, w_{n}$ in $L^{2}(Q)$. Of course this is impossible because the eigenvalues $\mu_{1}, \mu_{2}, \ldots, \mu_{n}$ are simple, due to our assumption on irrationality of $\ell_{1}^{2} \ell_{2}^{-2}$. This completes the proof of Theorem 2.1. 


\section{Asymptotic analysis of the spectrum for composite medium}

\subsection{Problem with periodic coefficients in half-planes}

Let us define the new coefficient function

$$
\mathbf{a}^{\varepsilon}(x)= \begin{cases}a^{\varepsilon}\left(x_{1}-h, x_{2}\right), & x_{1}>0, \\ a^{\varepsilon}\left(x_{1}+h, x_{2}\right), & x_{1}<0,\end{cases}
$$

where $h \in\left(0, \ell_{1}\right)$ and the numbers $\ell_{j}$ are rescaled as $\ell_{2}=1 / 2$ and $\ell_{1}>1 / 2$. Here, we realize the reflection on Fig. 1.2,b. The geometric setting is simple enough so that the function (3.1) can be made smooth by a proper choice of the old one (1.7) inside the thin frame $Q_{2 \varepsilon} \backslash Q_{\varepsilon}$ (for example, $a^{\varepsilon}$ is independent of $x_{1} \in\left(-\ell_{1}+3 \varepsilon, \ell_{1}+3 \varepsilon\right)$ ). The difference between (1.6) and the new equation

$$
-\operatorname{div}\left(\mathbf{a}^{\varepsilon}(x) \nabla_{x} \mathbf{u}^{\varepsilon}(x)\right)=\boldsymbol{\lambda}^{\varepsilon} \mathbf{u}^{\varepsilon}(x), \quad x \in \mathbb{R}^{2},
$$

is the loss of the periodicity in the $x_{1}$-direction due to the coefficient (3.1): as indicated in Fig.1.2.b), the two half-planes, which are paved with identical rectangles of size $2 \ell_{1} \times 2 \ell_{2}$, are now separated by a column of rectangles of size $2\left(\ell_{1}+h\right) \times 2 \ell_{2}$.

Let us denote by $\mathcal{T}(\varepsilon)$ the self-adjoint operator of the problem $(\underline{3.2})$, defined in the same way as in Section 1.2.

\subsection{Partial FBG-transform and model problem in the unit strip.}

Let us examine the spectrum of the problem (3.2). To this end, we apply the partial FBGtransform

$$
\mathbf{u}^{\varepsilon}(x) \mapsto \mathbf{U}^{\varepsilon}(x ; \zeta)=\frac{1}{\sqrt{2 \pi}} \sum_{k \in \mathbb{Z}} e^{-i \zeta k} \mathbf{u}^{\varepsilon}\left(x_{1}, x_{2}+k\right), \quad \zeta \in[0,2 \pi]
$$

and arrive at the model problem in the horizontal unit strip $\Pi=\mathbb{R} \times(-1 / 2,1 / 2)$

$$
\begin{aligned}
& -\operatorname{div}\left(\mathbf{a}^{\varepsilon}(x) \nabla_{x} \mathbf{U}^{\varepsilon}(x ; \zeta)\right)=\Lambda^{\varepsilon} \mathbf{U}^{\varepsilon}(x, \zeta), \quad x \in \Pi, \\
& \mathbf{U}^{\varepsilon}\left(x_{1}, \frac{1}{2} ; \zeta\right)=e^{i \zeta} \mathbf{U}^{\varepsilon}\left(x_{1},-\frac{1}{2} ; \zeta\right), \quad x_{1} \in \mathbb{R}, \\
& \partial_{x_{2}} \mathbf{U}^{\varepsilon}\left(x_{1}, \frac{1}{2} ; \zeta\right)=e^{i \zeta} \partial_{x_{2}} \mathbf{U}^{\varepsilon}\left(x_{1},-\frac{1}{2} ; \zeta\right), \quad x_{1} \in \mathbb{R} .
\end{aligned}
$$

It is known, see [19, Thm. 5], that for any fixed $\zeta$ the essential spectrum of the problem (3.3) is the union of the spectral bands

$$
\mathbf{B}_{k}^{\varepsilon}(\zeta)=\left\{\Lambda_{k}^{\varepsilon}\left(\eta_{1}, \zeta\right): \eta_{1} \in[-\pi, \pi]\right\}, \quad k \in \mathbb{N} .
$$

Moreover, there holds the relations $\mathbf{B}_{k}^{\varepsilon}(\zeta) \subset \beta_{k}^{\varepsilon}, k \in \mathbb{N}$.

The variational formulation of the problem (3.3) is

$$
\left(\mathbf{a}^{\varepsilon} \nabla_{x} \mathbf{U}^{\varepsilon}, \nabla_{x} \mathbf{V}\right)_{\Pi}=\Lambda^{\varepsilon}(\zeta)\left(\mathbf{U}^{\varepsilon}, \mathbf{V}\right)_{\Pi} \quad \forall \mathbf{V} \in \mathbf{H}_{\zeta}^{1}(\Pi),
$$

where $\mathbf{H}_{\zeta}^{1}(\Pi)$ is the space of functions in $H^{1}(\Pi)$ satisfying the first quasiperiodicity condition in $(3.3)$. 


\subsection{Asymptotics of eigenvalues and trapped modes in the strip.}

The appearance of the longer rectangle $\mathbf{Q}=\mathbf{Q}_{1}=\left(-\ell_{1}-h, \ell_{1}+h\right) \times(-1 / 2,1 / 2)$ in the paving of $\Pi$ leads to the new limit problem

$$
-\Delta_{x} \mathbf{w}(x)=\boldsymbol{\mu} \mathbf{w}(x) \text { in } \mathbf{Q}, \quad \partial_{\nu} \mathbf{w}(x)=0 \text { in } \partial \mathbf{Q} .
$$

The first positive eigenvalue of this problem is $\boldsymbol{\mu}_{2}=\frac{\pi^{2}}{4}\left(\ell_{1}+h\right)^{-2}$, corresponding to the eigenfunction $\mathbf{w}_{2}(x)=\left(\ell_{1}+h\right)^{-1 / 2} \sin \left(\pi\left(\ell_{1}+h\right)^{-1} x_{1} / 2\right)$. Notice that (2.10) implies

$$
\boldsymbol{\mu}_{2} \in\left(\mu_{1}, \mu_{2}\right) \text {. }
$$

Theorem 3 For any $\zeta \in[-\pi, \pi]$ there exist positive $\varepsilon_{2}$ and $\mathbf{c}_{2}$ such that the problem (3.3) has an eigenvalue $\boldsymbol{\Lambda}_{2}^{\varepsilon}(\zeta)$ satisfying the inequality

$$
\left|\boldsymbol{\Lambda}_{2}^{\varepsilon}(\zeta)-\boldsymbol{\mu}_{2}\right| \leq \mathbf{c}_{2} \varepsilon^{\gamma-1 / 2} \quad \forall \varepsilon \in\left(0, \boldsymbol{\varepsilon}_{2}\right) .
$$

Proof. We set $\mathbf{W}(x)=\mathbf{X}^{\varepsilon}(x) \mathbf{w}\left(\mathbf{A}_{\varepsilon}^{-1} x\right)$, where $\mathbf{X}^{\varepsilon}$ is a smooth cut-off function such that

$$
\mathbf{X}^{\varepsilon}=1 \text { in } \mathbf{Q}_{\varepsilon}, \quad \mathbf{X}^{\varepsilon}=0 \text { in } \mathbf{Q} \backslash \mathbf{Q}_{\varepsilon / 2}, \quad\left|\nabla_{x} \mathbf{X}^{\varepsilon}\right| \leq C_{\mathbf{X}} \varepsilon^{-1} \text { in } \mathbf{Q},
$$

and $\mathbf{A}_{\varepsilon} x=\left(\left(1-2 \varepsilon\left(\ell_{1}+h\right)^{-1}\right) x_{1},\left(1-2 \varepsilon \ell_{2}^{-1}\right) x_{2}\right)$. It is enough to estimate

$$
\sup \left|\left(\mathbf{a}^{\varepsilon} \nabla_{x} \mathbf{w}, \nabla_{x} \mathbf{v}\right)_{\Pi}-\boldsymbol{\mu}(\mathbf{W}, \mathbf{v})_{\Pi}\right|=\sup \left|\left(\mathbf{a}^{\varepsilon} \nabla_{x} \mathbf{W}, \nabla_{x} \mathbf{v}\right)_{\mathbf{Q}}-\boldsymbol{\mu}(\mathbf{W}, \mathbf{v})_{\mathbf{Q}}\right|
$$

where the supremum is computed over the unit ball of the Hilbert space $\mathbf{H}_{\zeta}^{1}(\Pi)$ with the scalar product

$$
\langle\mathbf{u}, \mathbf{v}\rangle_{\Pi, \varepsilon}=\left(\mathbf{a}^{\varepsilon} \nabla_{x} \mathbf{u}, \nabla_{x} \mathbf{v}\right)_{\Pi}+(\mathbf{u}, \mathbf{v})_{\Pi} .
$$

This can be done repeating word by word our arguments in the second part of the proof of Theorem 2.1 in Section 2.4

Comparing formulas (3.6), (3.5) and (2.13), we see that if $\varepsilon$ is small enough, the spectrum $\sigma\left(\mathcal{T}(\varepsilon)\right.$ ) of the problem (3.2) contains, in addition to the spectral bands $\beta_{k}^{\varepsilon}$ of the spectrum $\sigma\left(\mathcal{T}^{0}(\varepsilon)\right)$, at least one spectral band

$$
\mathbf{B}_{2}^{\varepsilon}=\left\{\Lambda_{2}^{\varepsilon}(\zeta): \zeta \in[-\pi, \pi]\right\}
$$

which does not intersect the set $\sigma\left(\mathcal{T}^{0}(\varepsilon)\right)$. This observation gives a negative answer to the question (1.1) in Section 1.1.

Theorem 4 There exists positive $\varepsilon_{0}$ such that, for any $\varepsilon \in\left(0, \varepsilon_{0}\right)$, the spectrum $\sigma_{\mathrm{ess}}(\mathcal{T}(\varepsilon))$ of the problem (3.2) contains the spectral band (3.7) which does not intersect the spectrum $\sigma_{\text {ess }}\left(\mathcal{T}^{0}(\varepsilon)\right)$ of the problem (1.6).

It is quite obvious that using the techniques presented above one could prove more comprehensive results than Theorem 3. Indeed, many of the open spectral gaps between bands $\beta_{k}^{\varepsilon}$ apparently contain eigenvalues of the limit problem (3.4). Each of these isolated eigenvalues gives rise again for a small $\varepsilon$ to an eigenvalue of the problem (3.3) and thus also to an additional spectral band of the problem (3.2). However, for the sake of the shortness of the paper we refrain from going into the detailed proofs, although we are convinced that a more complete asymptotic description of the eigenvalues of the problem (3.3) would not require new ideas in addition to those given above. 


\section{Concluding remarks}

The existence of Rayleigh waves [2] travelling along interfaces in piecewise homogeneous elastic solids is well-known, cf. [4, 3] and others. Such waves do not exist in the case of scalar differential equations, the piecewise constant coefficients of which have jumps at a straight line of the plane. However, the example constructed above shows that scalar second order equations with periodic coefficients may have propagating waves localized near infinite rows and columns of foreign inclusions. This was already predicted in [1].

Of course, changing the roles of coordinate axis as indicated in Fig.1.2. c) provides a row of bigger rectangles $\mathbf{Q}_{2}$ and also new spectral bands in the same way as in Section 3. Moreover, according to [1], these bands are preserved in the spectra, if the open waveguides containing a full row or column of rectangles are replaced by the corresponding semi-infinite open waveguides. Combining both of these constructions, we can create $\mathrm{X}$-, $\mathrm{T}$ - and $\mathrm{Y}$-shaped waveguides, which support propagating localized waves, cf. [1]. We also mention the papers [20, 21, 22 with other examples of localized propagating waves.

Let us consider the $\mathrm{X}$-shaped open waveguide in Fig.1.2. d), which contains the rectangle $\mathbf{Q}_{12}=\left\{x:\left|x_{1}\right|<\ell_{1}+h_{1},\left|x_{2}\right|<\ell_{2}+h_{2}\right\}$. The numbers $h_{j} \in\left(0, \ell_{j}\right)$ can be chosen such that the smallest positive eigenvalues of the Neumann problems (3.4) in $\mathbf{Q}_{j}, j=1,2$, and that of the problem (2.8), (2.9) in $Q$ can be ordered as follows:

$$
0<\frac{\pi^{2}}{\left(\ell_{1}+h_{1}\right)^{2}}<\frac{\pi^{2}}{\left(\ell_{2}+h_{2}\right)^{2}}<\frac{\pi^{2}}{\ell_{1}^{2}} .
$$

In addition, if $\ell_{1}<\sqrt{3}\left(\ell_{2}+h_{2}\right)$, then $h_{j}$ can still be adjusted to obtain

$$
\frac{\pi^{2}}{\left(\ell_{2}+h_{2}\right)^{2}}<\boldsymbol{\mu}_{1,2}=\frac{\pi^{2}}{\left(\ell_{1}+h_{1}\right)^{2}}+\frac{\pi^{2}}{\left(\ell_{2}+h_{2}\right)^{2}}<\frac{\pi^{2}}{\ell_{1}^{2}},
$$

where $\boldsymbol{\mu}_{1,2}$ is the Neumann eigenvalue for $-\Delta$ in $\mathbf{Q}_{12}$ corresponding to the eigenfunction

$$
\sin \left(\frac{\pi x_{1}}{\ell_{1}+h_{1}}\right) \sin \left(\frac{\pi x_{2}}{\ell_{2}+h_{2}}\right) .
$$

Now consider the spectral problem (3.2), where the coefficient $\mathbf{a}^{\varepsilon}$ is related to the $\mathbf{X}$-shaped open waveguide of Fig.[1.2 d). According to [1] and the conclusions in Sections 2 and 3, the first four spectral bands of this problem lie in the $c \varepsilon^{\gamma-1 / 2}$-neighbourhood of the points (4.1), although $\boldsymbol{\mu}_{1,2}$ is not contained in these bands. Thus, our previous asymptotic constructions, estimates and arguments prove that there exists an isolated eigenvalue in the vicinity of the point $\boldsymbol{\mu}_{1,2}$.

Proposition 5 Let $\ell_{j}$ and $h_{j}, j=1,2$, be fixed to fulfil the relations (4.1) and (4.2). There exists $\varepsilon_{d}>0$ such that, for any $\varepsilon \in\left(0, \varepsilon_{d}\right)$, the discrete spectrum of the problem corresponding to the $\mathbf{X}$-shaped open waveguide in fig. 1.2, d, contains at least one eigenvalue $\lambda_{d}(\varepsilon)=\boldsymbol{\mu}_{1,2}+$ $O\left(\varepsilon^{\gamma-1 / 2}\right)$.

Recall that if $\varepsilon$ is small, we have shown the existence of many open spectral gaps, cf. for example the end of Section 2.3. It might be possible to find also other eigenvalues (of the 
problem related to Fig.1.2, d) inside these gaps, just by using the above scheme to locate them near suitable Neumann eigenvalues of the problem in $\mathbf{Q}_{12}$. However, the first couple of the positive eigenvalues $\mu_{j}=\pi^{2}\left(\ell_{j}+h_{j}\right)^{-2}, j=1,2$, coincides with the numbers in (4.1) and therefore we do not know if they are included in the corresponding spectral bands or not. In other words, to prove or disprove the existence of isolated eigenvalues near $\boldsymbol{\mu}_{j}$ one would need to construct higher order terms in the asymptotic expansions.

An example of an eigenvalue embedded into the continuous spectrum of an open waveguide in a double periodic medium does not yet exist in the literature. We conjecture that this could be done using the concept of enforced stability of embedded eigenvalues, [23, 24], although it would require a much more delicate asymptotic analysis.

\section{Acknowledgment}

F.B. was supported by grants 0.38.237.2014 and 6.57.61.2016 of St.Petersburg University, and by grant 15-01-02175 of RFBR; S.N. by grant 15-01-02175 of RFBR and by the Academy of Finland project 268973; G.C. is a member of GNAMPA of INDAM; J.T. was partially supported by the Väisälä Foundation of the Finnish Academy of Sciences and Letters.

\section{References}

[1] Cardone G., Nazarov S.A., Taskinen J., Spectra of open waveguides in periodic media. J. Funct. Anal. 269 (2015), 2328-2364.

[2] Lord Rayleigh, On the Maintenance of Vibrations by Forces of Double Frequency, and on the Propagation of Waves Through a Medium Endowed with a Periodic Structure. Phil. Mag. 24 (1887), 145-59.

[3] Shams, M., Ogden, R. W., On Rayleigh-type surface waves in an initially stressed incompressible elastic solid. IMA J. Appl. Math. 79 (2014), 360376.

[4] Kamotskii, I.V., Kiselev, A.P., An energy approach to the proof of the existence of Rayleigh waves in an anisotropic elastic half-space. Journal of Applied Mathematics and Mechanics 73 (2009), 464-470.

[5] Hempel, R., Lienau, K., Spectral properties of periodic media in the large coupling limit. Comm. Partial Differential Equations 25 (2000), 1445-1470.

[6] Hempel, R., Post, O., Spectral Gaps for Periodic Elliptic Operators with High Contrast: An Overview. Progress in Analysis, Proceedings of the 3rd International ISAAC Congress Berlin (2001), 577-587.

[7] Zhikov, V.V., On gaps in the spectrum of some elliptic operators in divergent form with periodic coefficients. St. Petersb. Math. J. 16 (2005), 773-790.

[8] Nazarov, S.A., Gap in the essential spectrum of an elliptic formally self-adjoint system of differential equation. Differentsial'nye Uravneniya. 46, (2010), 726-736; English transl.: Differential equations 46 (2010), 730-741. 
[9] Birman, M.S, Solomyak, M.Z., Spectral theory of self-adjoint operators in Hilber space. Reidel Publ. Company, Dordrecht, 1986.

[10] Reed, M., Simon, B., Methods of Modern Mathematical Physics Vol I, Functional analysis. Second edition. Academic Press, Inc., New York, 1980.

[11] Gelfand, I.M., Expansion in characteristic functions of an equation with periodic coefficients. Dokl. Akad. Nauk SSSR 73 (1950), 1117-1120 (in Russian).

[12] Davies, E. B., Simon, B., Scattering theory for systems with different spatial asymptotics on the left and right. Comm. Math. Phys. 63 (1978), 277-301.

[13] Kuchment, P., Floquet theory for partial differential equations. Uspekhi Mat. Nauk 37 (1982), 3-52; English transl: Russ. Math. Surveys 37 (1982) 1-60.

[14] Skriganov, M.M., Geometric and arithmetic methods in spectral theory of multidimensional periodic operators. Trudy Mat. Inst. Steklov, 171 (1985), 3-122.

[15] Kuchment, P., Floquet theory for partial differential equations. Birkhäuser Verlag, Basel, 1993.

[16] Reed, M., Simon, B., Methods of modern mathematical physics. Vol. IV. Analysis of operators. Academic Press, New York-London, 1978.

[17] Kato, T., Perturbation theory for linear operators. Die Grundlehren der mathematischen Wissenschaften, 132 Springer-Verlag New York, 1966.

[18] Rudin, W. Functional Analysis. MacGraw-Hill, Inc., New York 1991.

[19] Nazarov, S.A., Elliptic boundary value problems with periodic coefficients in a cylinder. Izv. Akad. Nauk SSSR. Ser. Mat. 45 (1981), 101-112; English transl.: Math. USSR. Izvestija. 18 (1982), 89-98.

[20] Cardone, G., Durante, T., Nazarov, S.A., The spectrum, radiation conditions and the Fredholm property for the Dirichlet Laplacian in a perforated plane with semiinfinite inclusions. J. Differential Equations 263 (2) (2017), 1387-1418.

[21] Nazarov, S. A., Open waveguides in a thin Dirichlet lattice: II. Localized waves and radiation conditions. Comput. Math. Math. Phys. 57 (2) (2017), 236-252.

[22] Cardone, G., Khrabustovskyi, Spectrum of a singularly perturbed periodic narrow waveguide. J. Math. Anal. Appl. 454 (2) (2017), 673-694.

[23] Nazarov, S.A., Asymptotic expansions of eigenvalues in the continuous spectrum of a regularly perturbed quantum waveguide. Teoret. Mat. Fiz. 167 (2011), 239-263; Engl. transl.: Theoret. and Math. Phys. 167 (2011), 606-627.

[24] Nazarov, S. A., Forced stability of a simple eigenvalue in the continuous spectrum of a waveguide. Funktsional. Anal. i Prilozhen. 47 (2013), 37-53; Engl. transl.: Funct. Anal. Appl. 47 (2013), 195209. 